\title{
Cryptococcal Meningitis: Diagnosis and Management Update
}

\author{
Mahsa Abassi $^{1,2}$ • David R. Boulware ${ }^{1}$ Joshua Rhein ${ }^{1,2}$
}

Published online: 22 April 2015

(C) Springer International Publishing AG 2015

\begin{abstract}
Recent advances in the diagnosis and management of cryptococcal meningitis are promising and have been improving long-term survival. Point of care testing has made diagnosing cryptococcal meningitis rapid, practical, and affordable. Targeted screening and treatment programs for cryptococcal antigenemia are a cost-effective method for reducing early mortality on antiretroviral therapy (ART). Optimal initial management with amphotericin and flucytosine improves survival against alternative therapies, although amphotericin is difficult to administer and flucytosine is not available in middle- or low-income countries, where cryptococcal meningitis is most prevalent. Controlling increased intracranial pressure with serial therapeutic lumbar punctures has a proven survival benefit. Delaying ART initiation for 4 weeks after the diagnosis of cryptococcal meningitis is associated with improved survival. Fortunately, new approaches have been leading the way toward improving care for cryptococcal meningitis patients. New trials utilizing different combinations of antifungal therapy are reviewed, and we summarize the efficacy of different regimens.
\end{abstract}

Keywords HIV · AIDS · Cryptococcal meningitis · Immune reconstitution inflammatory syndrome $\cdot$ Review .

Antiretroviral therapy $\cdot$ Antifungal therapy

This article is part of the Topical Collection on Tropical Mycosis

Mahsa Abassi

abass004@umn.edu

1 Division of Infectious Diseases and International Medicine, Department of Medicine, University of Minnesota, MMC 250, 420 Delaware St SE, Minneapolis, MN 55455, USA

2 Infectious Disease Institute, Makerere University, Kampala, Uganda

\section{Introduction}

Cryptococcal meningitis remains a major cause of HIV-related mortality worldwide, with the largest burden of the disease in sub-Saharan Africa and South and Southeast Asia [1]. Inhospital acute mortality from cryptococcal meningitis continues to remain high, ranging between 30 and $50 \%$, even with antifungal therapy [2]. Despite declines in long-term mortality from the introduction of antiretroviral therapy (ART) [3], in low-income countries, ART distribution has not yet effectively reached all individuals needed to decrease the overall incidence of cryptococcal meningitis [4]. In addition to high mortality, cryptococcal meningitis has substantial morbidity. Survivors can suffer from irreversible blindness and deafness, as well as reversible neurocognitive impairments [5].

Fortunately, several recent innovations in the screening, diagnosis, and management of cryptococcal meningitis have shown how to improve care for patients in resource-limited settings. This review will focus on these innovations, providing an update in the field of cryptococcal diagnostics and management.

\section{Diagnosis of Cryptococcal Meningitis}

There are several modalities now available for the diagnosis of cryptococcal meningitis in HIV-infected persons [6]. Diagnosis centers upon detection by microscopy, culture, or antigen. The use of India ink staining remains a common diagnostic tool for identifying Cryptococcus in cerebrospinal fluid (CSF), yet the sensitivity of India ink microscopy is only $<86 \%[6,7]$. Although readily available, the use of the India ink as the sole means of diagnosis results in misdiagnosis in 1 of every 11 persons presenting with meningitis in Uganda [6]. India ink is particularly insensitive for low fungal burdens, 
which can be common in persons presenting early after symptom onset or those presenting on ART. The sensitivity of India ink decreases to $42 \%$ with fungal burdens of $<1000$ colonyforming units (CFU)/mL on quantitative CSF culture [6].

Culture is considered the gold standard for diagnosis of cryptococcal meningitis but has several disadvantages [8]. Fungal cultures require laboratory infrastructure, electricity, and trained technicians. Cultures can take up to 7 days to grow and need to be incubated for up to 10 days for a reliable quantitative count. Cultures can also produce false negative results when the fungal burden is low, though diagnostic yield can be improved using higher volumes of CSF. For example, using $100 \mu \mathrm{L}$ of undiluted CSF versus $10 \mu \mathrm{L}$ at five 1:10 dilutions improved the sensitivity from 82.4 to $94.2 \%$, with the minimum CFU needed for growth decreasing from 100 to $10 \mathrm{CFU} /$ $\mathrm{mL}$ [6]. Despite the infrastructural drawbacks of quantitative fungal cultures, quantification can provide an important measure of treatment response and is central in the diagnosis and differentiation of cryptococcal meningitis relapse versus paradoxical immune reconstitution syndrome (IRIS).

The detection of cryptococcal antigen ( $\mathrm{CrAg}$ ) in $\mathrm{CSF}$, serum, or plasma has become an essential diagnostic tool and should be performed on CSF for all patients with HIV with suspected meningitis or any central nervous system (CNS) symptoms. CSF testing should occur regardless of other cerebrospinal fluid parameters. Commercially available tests for the detection of $\mathrm{CrAg}$, either by latex agglutination or by enzyme immunoassay, have been available for several years [9]. The recent development of a CrAg lateral flow assay (LFA; Immy Inc., Norman, OK) has revolutionized cryptococcal meningitis diagnosis in resource-limited settings. The CrAg LFA is a point of care test that rapidly detects cryptococcal polysaccharide capsule using gold-conjugated anti-cryptococcal monoclonal antibodies directed against Cryptococcus neoformans [8]. Unlike latex agglutination, the CrAg LFA is stable at room temperature, does not require a cold chain or centralized laboratory, is inexpensive, and takes only $10 \mathrm{~min}$ to obtain results. The CrAg LFA also has slightly better sensitivity than latex agglutination or enzyme immunoassay and is more sensitive at detecting lower CSF antigen levels [6, 9].

Jarvis et al. tested the use of the CrAg LFA in the serum of 62 patients with a history of cryptococcal disease in South Africa [10]. A more recent multisite diagnostic study in Uganda and South Africa found the CrAg LFA to have a sensitivity of $99.3 \%$ and a specificity of $99.1 \%$ in CSF [6]. In a study published in South Africa, the sensitivity of the CrAg LFA in CSF was reported as only $91 \%$ [11]; however, when samples with a suspected high organism load underwent a 1:2 dilution prior to testing, sensitivity improved to $100 \%$, an incongruity attributed to the "hook effect," whereby a high cryptococcal antigen load interferes with the antigen-antibody complex of the assay producing false negative results.
The sensitivity of CrAg in blood is $\geq 99 \%$ when positive in CSF [12]. CrAg LFA can be tested in either serum or plasma [6]. The presence of serum antigenemia in any HIV-infected patient with central nervous system symptoms should provoke a lumbar puncture with measurement of opening intracranial pressure $[13,14]$. In a recent study in Uganda, there was perfect agreement between fingerstick whole blood, serum, and plasma CrAg LFA suggesting that testing from fingerstick whole blood is a viable option for detecting antigen $\mathrm{CrAg}$, particularly in settings where phlebotomy is not available, or in patients with difficult venous access [15]. The CrAg LFA has also been evaluated in both urine and saliva, but agreement with serum LFA was not sufficient to recommend routing screening using these fluids [16, 17].

The use of semiquantitative CrAg LFA titers has been demonstrated to correlate with pretreatment quantitative cultures but has not been found to be useful for monitoring treatment response. CrAg titers can also be used as a prognostic marker as titers $>1: 1024$ are associated with greater mortality at 2 and 10 weeks [18]. However, performing CrAg titers can be labor intensive, requiring extra diluent, and increasing the cost.

In addition to these primary modalities available for diagnosing cryptococcal meningitis, non-specific markers of fungal infection should raise a concern for cryptococcosis in immune-compromised patients and may play a useful role in prognosis and classification. High levels of $(1 \rightarrow 3)-\beta$-D-glucan (BDG), for instance, were observed in the CSF of a recent cohort of Ugandan and South African HIV-infected patients with cryptococcal meningitis [19]. In this study, the Fungitell BDG assay (Associates of Cape Cod, Inc., Falmouth, Massachusetts) was found to have a sensitivity of $89 \%$ and a specificity of $85 \%$ as compared to the CrAg LFA. Detectable levels of BDG in CSF were found to correlate with quantitative fungal cultures, and BDG values of $>500 \mathrm{pg} / \mathrm{mL}$ were associated with increased mortality. BDG was found to rapidly decline with the start of antifungal therapy, falling $\sim 50 \%$ after 4 days, unlike CrAg titers, which may continue to persist long after the completion of antifungal therapy. Although BDG is inferior to CrAg LFA in regard to diagnosis of firstepisode cryptococcal meningitis, potential applications include to use as a prognostic indicator for mortality, to monitor treatment response, and to help differentiate culture-positive relapse (i.e., positive BDG) versus paradoxical immune reconstitution inflammatory syndrome (IRIS) with negative BDG.

Similarly, PCR-based diagnosis of cryptococcal meningitis has not been widely developed given the high sensitivity, wide availability, and low cost of $\mathrm{CrAg}$ testing. However, in certain populations, such as those presenting with recurrent or persistent symptoms of meningitis, PCR testing may provide a clinically useful adjunct to traditional testing. In one recent study from Uganda, the CSF of $39 \mathrm{HIV}$-infected persons with suspected cryptococcal meningitis were evaluated with the FilmArray System (BioFire Diagnostics, Salt Lake City, UT) 
using amultiplex meningitis/encephalitis PCR panel [20]. The FilmArray system was able to detect Cryptococcus with $100 \%$ sensitivity and specificity, was able to differentiate between C. neoformans and Cryptococcus gattii, and was able distinguish between relapse and paradoxical IRIS.

\section{Management of Cryptococcal Meningitis}

The management of cryptococcal meningitis is divided into three phases: (1) induction, (2) consolidation, and (3) maintenance therapy (Table 1). The goal of induction therapy is the rapid sterilization of cerebrospinal fluid. A quantitative measure of this is the rate of yeast clearance per milliliter of CSF per day. This quantitative clearance is termed early fungicidal activity (EFA). Slower rates of fungal clearance have been shown to be associated with increased mortality at both 2 and 10 weeks [21].

\section{Induction Antifungal Therapy}

Current guidelines recommend 2 weeks of amphotericin B $(0.7-1.0 \mathrm{mg} / \mathrm{kg}$ per day) intravenously in combination with flucytosine $100 \mathrm{mg} / \mathrm{kg} /$ day as the first-line therapy for treatment of cryptococcal meningitis [22] (Fig. 1). The efficacy of amphotericin B and flucytosine in improving survival was recently demonstrated in a landmark trial comparing three different induction therapies: (1) high-dose amphotericin B monotherapy for 4 weeks, (2) high-dose amphotericin B combined with flucytosine for 2 weeks, and (3) high-dose amphotericin B with high-dose fluconazole for 2 weeks $[23 \bullet \cdot]$. Combination therapy with amphotericin B and flucytosine was associated with a $\sim 40 \%$ lower hazard of mortality at 10 weeks. This effect persisted at 6 months and was associated with increased rates of fungal clearance as compared to 4 weeks of amphotericin monotherapy. Despite the superiority of combination therapy with amphotericin B and

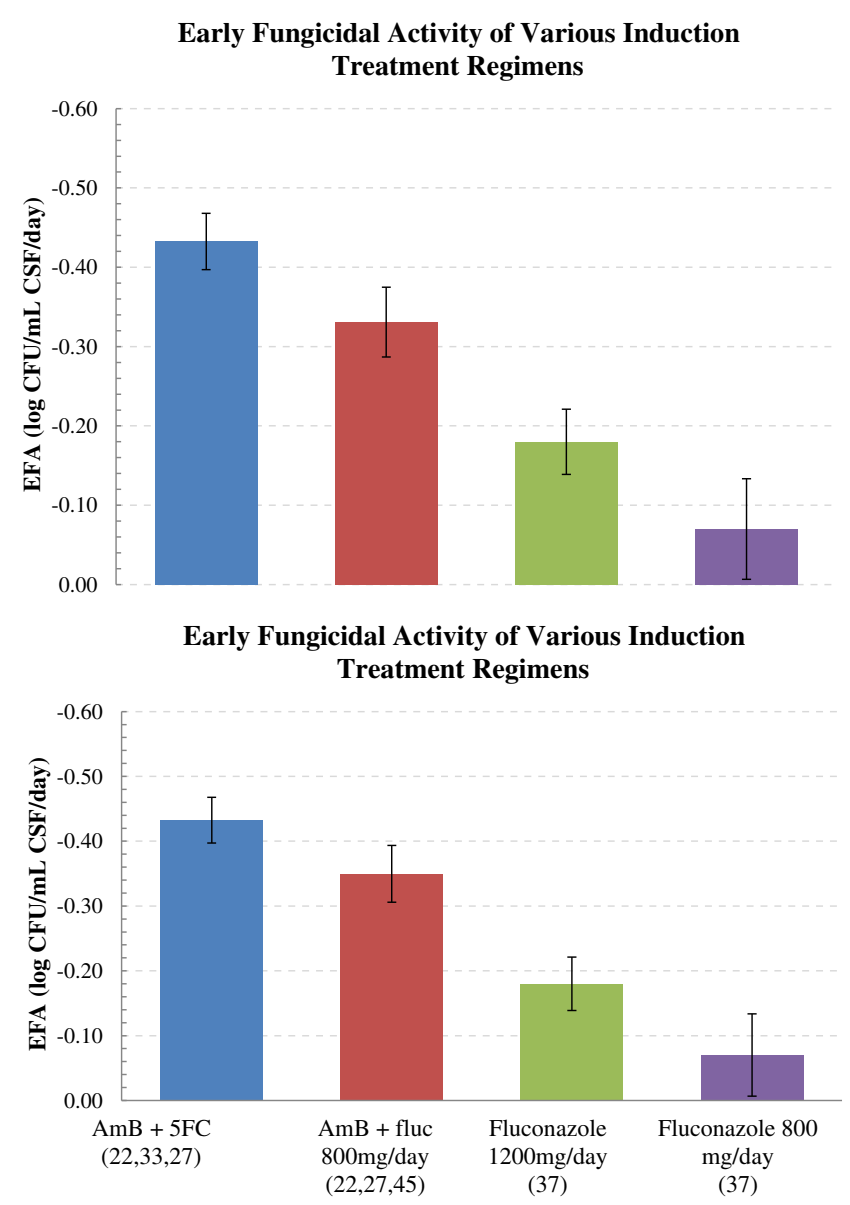

Fig. 1 Early fungicidal activity (EFA) of induction regimens for the treatment for cryptococcal meningitis, measured as $\log _{10}$ clearance of yeasts per milliliter of CSF per day in quantitative CSF culture. Values are the means with $95 \%$ confidence intervals as pooled from Table 2 (citations are in parenthesis). $A m B$ amphotericin, fluc fluconazole, $C F U$ colony-forming units, $C S F$ cerebrospinal fluid

flucytosine over alternative regimens, this regimen remains widely unavailable in most parts of the world with the highest burdens of disease. With only one manufacturer in 2014, the cost of flucytosine is US\$700/day for a $70-\mathrm{kg}$ adult. This

Table 1 Recommended treatment for cryptococcal meningitis in resource-limited settings

\begin{tabular}{|c|c|c|c|}
\hline Medication and dose & $1-2$ weeks $^{\mathrm{a}}$ & 12 weeks & 52 weeks \\
\hline \multicolumn{4}{|c|}{ Amphotericin $(0.7-1.0 \mathrm{mg} / \mathrm{kg} /$ day $)+$ second adjunctive agent $^{\mathrm{b}}$} \\
\hline Fluconazole $800-1200 \mathrm{mg}$ daily & Continue until CSF is known sterile ${ }^{c}$ & & \\
\hline Fluconazole 400 mg daily & & & c \\
\hline Fluconazole $200 \mathrm{mg}$ daily & & & Until CD4 $>200$ for $\geq 6$ months \\
\hline Treatment phase & Induction & Consolidation & Secondary prophylaxis \\
\hline
\end{tabular}

${ }^{a}$ Optimal duration of initial induction therapy is unknown. In resource-limited regions, the cost benefit is likely maximal for 1 week induction with amphotericin B at $1 \mathrm{mg} / \mathrm{kg} /$ day coupled with 4 weeks of fluconazole $1200 \mathrm{mg} \mathrm{kg} /$ day [23••]

${ }^{\mathrm{b}}$ Flucytosine (5FC) $100 \mathrm{mg} / \mathrm{kg} /$ day preferred where available, otherwise fluconazole at 800-1200 mg/day in divided doses. $\mathrm{KCl} 40-60 \mathrm{mEq} / \mathrm{day}$ should be given with amphotericin [30]

${ }^{\mathrm{c}}$ We recommend continuing fluconazole at $800-1200 \mathrm{mg} / \mathrm{day}$ until the CSF culture result is known to be sterile and ART has been initiated. Consider longer duration of consolidation therapy if CSF culture is positive at 2 weeks 
compares with a total treatment cost of US\$402 for 2 weeks of amphotericin with fluconazole [24]. Efforts within the medical community are currently underway to help address this flucytosine disparity $[25,26]$.

When flucytosine is unavailable, the combination of amphotericin B with fluconazole is recommended [22]. Pappas et al. demonstrated that the combination of amphotericin B with fluconazole $800 \mathrm{mg} /$ day had better long-term outcomes than amphotericin B and fluconazole $400 \mathrm{mg}$ /day or amphotericin alone [27]. Day et al. did not find a statistically different survival benefit between amphotericin with fluconazole $800 \mathrm{mg} /$ day and amphotericin alone, although fewer patients died in the combination arm [23••]. Loyse et al. did not find a statically significant difference in EFA of amphotericin B with fluconazole at $800-1200 \mathrm{mg} /$ day [28]. Therefore, current guideline favors the use of amphotericin B in combination with fluconazole $\geq 800 \mathrm{mg}$ / day when flucytosine is not available [22] (Table 2).

Amphotericin B is known to cause significant side effects including anemia, kidney insufficiency, hypokalemia, hypomagnesemia, and phlebitis. The administration of amphotericin requires inpatient hospitalization, intravenous administration, and a substantial nursing commitment. Therefore, administration of amphotericin over 14 days can not only become costly but also resource consuming. In a small study in Uganda, a short 5-day course of amphotericin B with high-dose fluconazole $1200 \mathrm{mg} /$ day had a superior EFA than either fluconazole at $800 \mathrm{mg} /$ day or $1200 \mathrm{mg}$ /day alone [29]. When flucytosine was added to a short, 7-day, course of amphotericin B plus high-dose fluconazole $1200 \mathrm{mg} /$ day, a greater EFA was observed than with amphotericin and fluconazole or fluconazole and flucytosine combinations [30]. Short course (5-7 days) amphotericin combined with high-dose fluconazole $1200 \mathrm{mg}$ /day is an alternative therapeutic option when 14 days of amphotericin is not feasible. Substantial life-threatening hypokalemia occurs during the second week, if not properly managed [31].

The use of voriconazole as a substitute for fluconazole in induction therapy has been studied and found to have similar EFA to amphotericin and fluconazole at both $800 \mathrm{mg} /$ day and $1200 \mathrm{mg} /$ day doses [28]. Although no benefit of voriconazole over fluconazole has been demonstrated for fluconazolesusceptible strains, a future role for newer antifungals for the treatment of cryptococcal meningitis might be anticipated, particularly in the context of increasing rates of fluconazole resistance, as costs come down, and worldwide availability of these drugs increase [32, 33]. Adjunctive interferon-gamma (INF- $\gamma$ ) has also been shown to be an effective component of combination induction therapy. Jarvis et al. demonstrated a $30 \%$ increased rate of clearance with 2 doses of adjunctive interferon-gamma than with standard therapy of amphotericin and flucytosine [34].

Infectious Diseases Society of America (IDSA) and WHO guidelines continue to recommend high-dose fluconazole monotherapy at $1200 \mathrm{mg} /$ day for $10-12$ weeks if amphotericin B and flucytosine are not available [22, 35, 36]. While clearly suboptimal compared to combination
Table 2 Trials comparing early fungicidal activity of induction treatment regimens for cryptococcal meningitis

\begin{tabular}{lrrrr}
\hline Induction regimen & \multicolumn{1}{l}{ EFA } & \pm SD & Number & Source \\
\hline Amphotericin & -0.31 & 0.15 & 99 & {$[23 \bullet \cdot]$} \\
Amphotericin + 5FC & -0.42 & 0.10 & 100 & \\
Amphotericin + fluconazole (800 mg/day) & -0.32 & 0.10 & 99 & \\
Amphotericin + 5FC & -0.41 & 0.22 & 21 & {$[28]$} \\
Amphotericin + fluconazole (800 mg/day) & -0.38 & 0.18 & 22 & \\
Amphotericin + fluconazole (1200 mg/day) & -0.41 & 0.35 & 23 & \\
Amphotericin + voriconazole & -0.44 & 0.20 & 13 & \\
Amphotericin (5 days) + fluconazole (1200 mg/day) & -0.30 & 0.11 & 30 & {$[29]$} \\
Amphotericin + 5FC & -0.49 & $\mathrm{NA}$ & 30 & {$[34]$} \\
Amphotericin + 5FC + INF- $\gamma$ & -0.64 & $\mathrm{NA}$ & 60 & \\
Amphotericin (7 days) + fluconazole (1200 mg/day) & -0.38 & 0.20 & 19 & {$[30]$} \\
Amphotericin (7 days) + fluconazole (1200 mg/day) + 5FC & -0.50 & 0.15 & 18 & \\
Fluconazole (1200 mg/day) & -0.18 & 0.11 & 30 & [38] \\
Fluconazole (800 mg/day) & -0.07 & 0.17 & 30 & \\
Amphotericin (1 mg/kg/day) & -0.48 & 0.28 & 49 & [39] \\
Fluconazole (400 mg/day) & -0.02 & 0.05 & 5 & \\
Amphotericin + fluconazole (800 mg/day) & -0.36 & 0.25 & 223 & [45••] \\
Amphotericin + fluconazole (800 mg/day) + sertraline & -0.44 & 0.39 & 113 & [49] \\
\hline
\end{tabular}

Amphotericin B deoxycholate 0.7 or $1 \mathrm{mg} / \mathrm{kg}$ /day or as indicated, voriconazole (300 mg twice daily; $400 \mathrm{mg}$ twice on day 1)

EFA early fungicidal activity ( $\log _{10} \mathrm{CFU} / \mathrm{mL} \mathrm{CSF} /$ day) calculated by linear regression except for Day et al. which is calculated with mixed regression model [23••], $5 F C$ flucytosine $(25 \mathrm{mg} / \mathrm{kg} 4$ times daily), INF- $\gamma$ interferongamma (100 $\mu \mathrm{g}$ subcutaneously, 2 or 6 doses) 
amphotericin therapy, fluconazole remains the only therapeutic option for the treatment of cryptococcal meningitis in much of the world, where amphotericin or flucytosine are unavailable. A recent study in Malawi demonstrated high morality ( $43 \%$ at 4 weeks) and treatment failure (77\% at 1 year) with the use of $800 \mathrm{mg} /$ day of fluconazole monotherapy for induction therapy [37]. Fluconazole doses of $1200 \mathrm{mg} /$ day for the first 2 weeks of induction therapy were associated with an increase in EFA as compared to $800 \mathrm{mg} /$ day, although no differences in mortality were seen at either 2 or 10 weeks [38].

\section{Consolidation and Maintenance Therapy}

Consolidation phase of therapy currently consists of fluconazole $400-800 \mathrm{mg} /$ day for at least 8 weeks [22]. Most guidelines recommend starting consolidation therapy after 2 weeks of induction therapy, though the start of consolidation therapy should be individualized based on patient response to induction therapy. In a study in Uganda, $56 \%$ of patients treated with amphotericin-based therapy had positive cultures at the end of 2 weeks [2]. Because fluconazole at $400 \mathrm{mg} / \mathrm{day}$ is fungistatic [39], a lumbar puncture and culture should be done at 2 weeks to demonstrate CSF sterility. When CSF sterility has been documented (often after 10-14 days of further culture incubation), the fluconazole dose should then be decreased from $800 \mathrm{mg} /$ day to $400 \mathrm{mg}$ /day. Guidelines support the use of longer durations of high-dose fluconazole throughout the consolidation phase if using suboptimal induction therapy; mainly, monotherapy with fluconazole or when CSF sterility has not been achieved [22].

After successful induction and consolidation therapy, culture-negative patients should be placed on fluconazole $200 \mathrm{mg} /$ day for maintenance therapy [22]. Recommendations for the use of long-term fluconazole stems from observations made in the pre-ART era of high relapse rates when therapy was discontinued [40]. Fluconazole $200 \mathrm{mg}$ /day was found to be superior when compared to weekly intravenous administration of amphotericin B in preventing cryptococcal meningitis relapse [41]. Comparison of fluconazole to itraconazole reproduced similar results [42]. Historically, this switch to secondary prophylaxis was made after 8 weeks of consolidation therapy (i.e., 10 weeks after diagnosis). Our own experience prefers longer consolidation therapy, switching to secondary prophylaxis after 2-3 months of ART, which allows for time for immune recovery to occur on ART.

Secondary prophylaxis can be safely discontinued in patients on ART and with undetectable HIV RNA levels for greater than 3 months, with CD 4 cell counts $\geq 100$ cells $/ \mu \mathrm{L}$ [22]. When HIV viral load testing is unavailable, the WHO recommends continuation of maintenance therapy for 1 year and discontinuation if CD4 counts are $>200$ cells $/ \mu \mathrm{L}$ [35]. Fluconazole maintenance therapy should be reinstituted in patients demonstrating immunologic failure, ART interruptions, or a fall in CD4 counts to below 100 cell/ $\mu \mathrm{L}$ [22].

\section{Management of Amphotericin B-Related Toxicities}

Although amphotericin-based therapies for cryptococcal meningitis remain central for the treatment of cryptococcal meningitis, the side effects, cost of monitoring, storage needs, and nursing staff for administration may be a deterrent for use in low-resource facilities. Intravenous (IV) administration of amphotericin through a peripheral line often causes thrombophlebitis or peripheral venous thrombosis. IV lines should be routinely flushed and rotated every 3 days to minimize phlebitis. Amphotericin frequently causes infusion-related reactions, including fevers, rigors, and nausea. Adjunctive acetaminophen can be given for symptomatic management of infusion reactions with hydrocortisone reserved for severe reactions [43].

Acute kidney injury occurs with cumulative doses of amphotericin and is reversible with discontinuation. Administration of ample amounts (1-2 L of normal saline or high sodium chloride dose) of IV fluid while receiving amphotericin can help prevent renal insufficiency. Increasing fluid administration or alternate day dosing of amphotericin are options if there is a greater than twofold rise in serum creatinine from baseline [35].

Potassium and magnesium wasting occur universally with amphotericin administration and requires close electrolyte monitoring and supplementation to prevent life-threatening hypokalemia. Electrolyte wasting begins after 5 days of amphotericin therapy. If electrolyte monitoring is not available, a standardized protocol for electrolyte supplementation and replacement can be instituted. The implementation of a standardized electrolyte protocol reduces the incidence of severe, life-threatening hypokalemia and improves survival [31]. Electrolyte management with amphotericin is recognized as an essential part of the package of care in World Health Organization cryptococcal treatment guidelines [35].

\section{Identifying Novel Antifungal Agents for Cryptococcal Meningitis}

There are several shortcomings to drugs currently considered standard of care for the treatment of cryptococcal meningitis, as outlined above. Fluconazole is primarily fungistatic, and although it penetrates well into the CNS, even at high doses, it has relatively poor fungal clearance. Fluconazole is both dose dependent and inoculum dependent [44]. Amphotericin B has better efficacy but substantial toxicity [45••], needs to be administered intravenously, and is not readily available in low-resource countries, even though amphotericin is on the WHO Essential Medication list. Flucytosine is currently not available in Asian and African countries that bear the largest burden of cryptococcal meningitis and has been associated 
with hematologic toxicity. There has therefore been a push for the development of new therapies that are (1) orally bioavailable, (2) low cost, (3) associated with low toxicity, and (4) fungicidal. While the antifungal activity of many novel compounds is currently being examined, the immediate and critical need has led some researchers to evaluate known compounds with the hope of identifying agents that can be repurposed as new antifungals [46].

The antidepressant sertraline has been found to have potent fungicidal activity against Cryptococcus both in vitro and in vivo animal models [47] (Table 2). Sertraline reaches $\sim 20$ fold higher concentration in the brain as well as $\sim 65$-fold higher concentrations in the lung than in the blood [48], and sertraline has a bidirectional synergistic effect with fluconazole [47, 49]. There is currently a phase III randomized clinical trial underway to investigate 18 -week survival of adjunctive sertraline to standard therapy for the treatment of cryptococcal meningitis (ASTRO-CM, clinicaltrials.gov. NCT01802385). Other drugs that have demonstrated to have anti-cryptococcal activity include astemizole, polymixin B, miltefosine, tamoxifen, amiodarone, and thioridazine, although their clinical role for the treatment of human cryptococcosis has yet to be tested [46].

\section{Management of Intracranial Pressure}

Elevated intracranial pressure (ICP) is defined as CSF pressure $\geq 25 \mathrm{~cm} \mathrm{H}_{2} \mathrm{O}$ and is a common complication of cryptococcal meningitis. The mechanism of elevated ICP is primarily due to a failure of CSF resorption via the arachnoid villa due to the physical obstruction by cryptococcal polysaccharide capsule [50]. The degree of elevated ICP is correlated with the amount of organisms found in the arachnoid granulations and size of the capsule $[50,51]$. Cerebral edema can be a mechanism of increased ICP in HIV-negative persons who may have a much greater degree of CSF inflammation [52, 53]. Elevated ICP is most often characterized by headaches, vomiting, papilledema, reduction of visual acuity, blindness, cranial nerve palsy's (most commonly cranial nerve VI), confusion, altered mental status, and coma [2, 54, 55]. Significantly elevated ICP that is not addressed causes increased 1014-day mortality [56, 57•, 58]. Current guidelines strongly recommend the aggressive management of ICP [22]. Management should consist of a baseline lumbar puncture, measurement of elevated ICP, CSF drainage if pressures are $\geq 25 \mathrm{~cm}$ $\mathrm{H}_{2} \mathrm{O}$ or in instances when symptoms are consistent with elevated ICP, and daily lumbar punctures until pressures have decreased or symptoms have resolved [22, 35, 59]. These high-income country guidelines are not very realistic in resource-limited settings and typically ignored. Rolfes et al. demonstrated the importance of aggressive ICP management with a $69 \%$ relative survival benefit with at least one therapeutic lumbar puncture (LP), and this survival benefit was irrespective of initial ICP [57•]. Specifically, the association was observed regardless of opening pressure at baseline. Those with normal opening pressures at baseline who did not receive a repeat therapeutic LP (which would be per guidelines) had higher 10-day mortality than those who received an additional therapeutic LP [60]. Similar results were seen in Tanzania, where a strict protocol for serial lumbar punctures decreased 30-day mortality from 75 to $46 \%$ [61]. In resourcelimited settings where manometers are not available, IV tubing or non-invasive methods such as handheld tonometers or ultrasound to measure intraocular pressure can be used as a surrogate for measuring ICP $[61,62]$. With persistently elevated ICP, ventriculoperitoneal shunts can be used when conservative measures have failed [63]. Other methods of decreasing ICP such as acetazolamide, mannitol, or corticosteroids should not be routinely used [56].

If opening pressures cannot be measured, we recommend (1) CrAg LFA screening by fingerstick prior to lumbar puncture; (2) for CRAG+ persons, presumptive removal of $20 \mathrm{~mL}$ of CSF at diagnosis; (3) repeat LP in 48-72 $\mathrm{h}$ with measurement of ICP with intravenous tubing or removal of $20 \mathrm{~mL}$; and (4) consideration of lumbar punctures at 7 and 14 days.

\section{Optimal Timing of ART Initiation}

The timing of ART initiation is an important consideration for persons with cryptococcal meningitis as with advanced immunosuppression, people are at high risk of AIDS progression and death [64]. However, ART initiation should be balanced against the risk for the development of paradoxical immune reconstitution inflammatory syndrome (IRIS). A consensus case definition of cryptococcal paradoxical IRIS defines the clinical syndrome as one occurring after treatment of the initial cryptococcal meningitis followed by ART initiation with subsequent clinical deterioration manifesting as one of the following: aseptic meningitis, intracranial lesions, lymphadenopathy, pneumonitis or pulmonary nodules, or cutaneous soft tissue lesions [65]. The reported incidence of paradoxical cryptococcal IRIS is highly variable in incidence, ranging between 8 and $49 \%$, presenting as soon as 4 days and up to 6 years after ART initiation, and carrying a mortality rate of $0-36 \%$ [65-67]. Better microbiologic therapy and achieving CSF sterility is a key principle at reducing the risk of IRIS [68]. In Uganda, we decreased the incidence of CNS events from 30 to $13 \%$ by adding fluconazole $800 \mathrm{mg} /$ day to the induction therapy and continuing for 4-6 weeks until ART initiation [45••, 69].

A landmark randomized trial conducted in Uganda and South Africa found a $15 \%$ higher 26 -week mortality in individuals initiating ART at 1-2 weeks from diagnosis as compared with those initiating ART 4-6 weeks after meningitis diagnosis [45••]. Three other smaller trials showed increased risk of death with earlier ART [70], increased risk of IRIS [71], and no differences [64]. Timing of ART remains 
somewhat controversial as three African trials showed increased harm with earlier ART whereas a USA-based trial showed no difference. No trial has shown any benefit of earlier ART with cryptococcal meningitis. Based on randomized clinical trial data $[45 \cdot \bullet]$, we recommend completion of induction therapy, a verification that the CSF culture at 14 days is sterile, with an aim to initiate ART at approximately 4 weeks. Persons lacking CSF pleocytosis are at high risk of IRIS [72], and these persons in particular are at higher risk of death when starting ART at $<2$ weeks [45••].

Finally, increased ART availability in resource-limited settings, coupled with a lack of pre-ART CrAg screening, has led to a greater proportion of patients developing cryptococcal meningitis after initiating ART. In places where cryptococcal meningitis once manifested primarily as an AIDS-defining illness in ART-naïve individuals, the occurrence of cryptococcal meningitis after initiating ART has now become common. In two cohorts from Uganda and South Africa, individuals already receiving ART at time of diagnosis had higher CD4 counts and lower fungal burdens, but outcomes were not improved $[73,74]$. Furthermore, individuals in the Ugandan study who developed cryptococcal meningitis within 14 days of initiating ART had significantly higher 2-week mortality (43\% compared with those on ART for 15 days to 4 months $(16 \%),>4$ months $(10 \%)$, or ART naïve $(25 \%) ; p=.05)$. This study underscores the detrimental effect of immune recovery in the setting of an untreated CNS infection and the importance of pre-ART cryptococcal antigen screening to prevent cryptococcal meningitis occurring early after ART initiation $[59,75]$.

\section{Cryptococcal Meningitis Relapse}

Cryptococcal meningitis relapse, or microbiological relapse, is the recurrence of meningeal symptoms with recovery of organism on CSF culture [76]. Microbiological relapse must be distinguished from paradoxical immune reconstitution syndrome in which symptoms recur but CSF cultures are found to be sterile. In a South African study, fluconazole nonadherence was found to be the primary cause of relapse. Therapy for cryptococcal meningitis relapse consists of reinitiating induction therapy with amphotericin $(1 \mathrm{mg} / \mathrm{kg} /$ day $)$ and higher-dose fluconazole (800-1200 mg/day) [22]. Voriconazole and INF- $\gamma$ have been used in case reports for salvage therapy in cases of cryptococcal meningitis refractory to standard therapy [77].

Cryptococcal meningitis relapse should be differentiated from persistent infection, or treatment failure. Whereas relapse occurs after documentation of sterile cultures, a person with persistent infection will continue to have positive cultures after 4 weeks of standard therapy, at effective doses. Susceptibility testing should be done on isolates to assess fluconazole resistance if persistent infection is suspected, and brain imaging should be considered to rule out cryptococcoma. Fluconazole resistance should be considered whenever the minimum inhibitory concentration (MIC) is $\geq 64 \mu \mathrm{g} / \mathrm{mL}$ [76].

\section{Diagnosis and Treatment of IRIS}

The presentation of recurrent symptomatic meningitis after the treatment of first episode of cryptococcal meningitis and post ART initiation should raise concern for disease relapse, treatment failure, or development of paradoxical IRIS. Symptomatic relapse may be secondary to either persistent infection due to fluconazole resistance, ineffective primary therapy, or presence of a cryptococcoma, whereas microbiological relapse has been shown to be mainly due to non-adherence of secondary fluconazole prophylaxis [76]. Distinguishing from treatment failure/relapse and paradoxical IRIS can be difficult, and the two entities are not always mutually exclusive. A positive cryptococcal culture, virologic failure, and lower CSF inflammatory profile support the diagnosis of cryptococcal meningitis relapse whereas a sterile culture and higher CSF WBC support the diagnosis of paradoxical IRIS [65, 72].

Management of IRIS, once the diagnosis has been made, includes management of elevated intracranial pressures with lumbar puncture and large volume drainage of CSF. Recommendations for therapeutic modalities are mainly based on expert opinion and clinical experience. For severe cases of IRIS with CNS complications, including increased intracranial pressure or neurological deterioration, the current IDSA guidelines recommends $0.5-1 \mathrm{mg} / \mathrm{kg}$ of prednisone or dexamethasone to be tapered over a 2-6-week period, although the duration of the taper may be individualized based on clinical status [22]. There have been several case reports documenting neurologic improvement with the use of thalidomide, a TNF- $\alpha$ inhibitor, in steroid-dependent or refractory cases of C-IRIS [78]. Adalimumab, a human monoclonal antibody that binds to TNF- $\alpha$, blocking its anti-inflammatory actions, demonstrated neurological improvement in a patient with IRISassociated cryptococcoma [79]. Both thalidomide and adalimumab were used after documented sterility of the CSF.

\section{Conclusion}

In conclusion, cryptococcal meningitis remains a prevalent opportunistic infection with high mortality and morbidity. The diagnosis of cryptococcal meningitis with CrAg LFA can help to detect disease early and rapidly without the need for any laboratory infrastructure. Newer diagnostics tools such as PCR assays and BDG measurements can aid in prognosis, monitoring treatment response, and diagnosing disease relapse versus IRIS. Combination amphotericin B and flucytosine is the best present induction therapy; however, 
the cost of flucytosine is prohibitive ( $\$ 700 /$ day). In the absence of flucytosine, concomitant fluconazole $800-1200 \mathrm{mg} /$ day can be used. After induction therapy, using enhanced consolidation therapy with fluconazole $800 \mathrm{mg}$ /day until documentation of CSF sterility and ART initiation should decrease the risk of persistent infection, disease relapse, or IRIS. Based on the timing of CSF sterility and immune recovery, longer duration of fluconazole consolidation therapy may be needed, often for 3-4 months. Crucial in the management of cryptococcal meningitis is aggressive control of elevated ICP by repeated lumbar punctures and drainage of CSF. A $70 \%$ relative decrease in 10-day mortality has been demonstrated by one additional therapeutic lumbar puncture (after a diagnostic lumbar puncture) irrespective of initial opening pressures. In ART-naïve persons, ART should be initiated 4-6 weeks after the diagnosis of cryptococcal meningitis. Earlier initiation of ART has been associated with increased mortality, particularly in those lacking CSF inflammation.

Acknowledgments The authors are supported by the National Institutes of Health, National Institute of Allergy and Infectious Diseases, and the Fogarty International Center (U01AI089244, T32AI055433, R25TW009345).

\section{Compliance with Ethics Guidelines}

Conflict of Interest Mahsa Abassi and David R. Boulware declare that they have no conflict of interest. Dr. Rhein reports grants from NIH, during the conduct of the study.

Human and Animal Rights and Informed Consent This article does not contain any studies with human or animal subjects performed by any of the authors.

\section{References}

Papers of particular interest, published recently, have been highlighted as:

- Of importance

•• Of major importance

1. Park BJ, Wannemuehler KA, Marston BJ, Govender N, Pappas PG, Chiller TM. Estimation of the current global burden of cryptococcal meningitis among persons living with HIV/AIDS. Aids. 2009;23(4):525-30.

2. Kambugu A, Meya DB, Rhein J, O'Brien M, Janoff EN, Ronald AR, et al. Outcomes of cryptococcal meningitis in Uganda before and after the availability of highly active antiretroviral therapy. Clin Infect Dis Off Publ Infect Dis Soc Am. 2008;46(11):1694-701.

3. Lortholary O, Poizat G, Zeller V, Neuville S, Boibieux A, Alvarez $\mathrm{M}$, et al. Long-term outcome of AIDS-associated cryptococcosis in the era of combination antiretroviral therapy. AIDS. 2006;20(17): 2183-91.

4. Jarvis JN, Boulle A, Loyse A, Bicanic T, Rebe K, Williams A, et al. High ongoing burden of cryptococcal disease in Africa despite antiretroviral roll out. Aids. 2009;23(9):1182-3.
5. Carlson RD, Rolfes MA, Birkenkamp KE, Nakasujja N, Rajasingham R, Meya DB, et al. Predictors of neurocognitive outcomes on antiretroviral therapy after cryptococcal meningitis: a prospective cohort study. Metab Brain Dis. 2014;29(2):269-79.

6. Boulware DR, Rolfes MA, Rajasingham R, von Hohenberg M, Qin $\mathrm{Z}$, Taseera $\mathrm{K}$, et al. Multisite validation of cryptococcal antigen lateral flow assay and quantification by laser thermal contrast. Emerg Infect Dis. 2014;20(1):45-53.

7. Kisenge PR, Hawkins AT, Maro VP, McHele JP, Swai NS, Mueller A, et al. Low CD4 count plus coma predicts cryptococcal meningitis in Tanzania. BMC Infect Dis. 2007;7(1):39.

8. Boulware D, Rhein. Prognosis and management of cryptococcal meningitis in patients with human immunodeficiency virus infection. Neurobehav HIV Med. 2012;45.

9. Temstet A, Roux P, Poirot JL, Ronin O, Dromer F. Evaluation of a monoclonal antibody-based latex agglutination test for diagnosis of cryptococcosis: comparison with two tests using polyclonal antibodies. J Clin Microbiol. 1992;30(10):2544-50.

10. Jarvis JN, Percival A, Bauman S, Pelfrey J, Meintjes G, Williams GN, et al. Evaluation of a novel point-of-care cryptococcal antigen test on serum, plasma, and urine from patients with HIV-associated cryptococcal meningitis. Clin Infect Dis Off Publ Infect Dis Soc Am. 2011;53(10):1019-23.

11. Lourens A, Jarvis JN, Meintjes G, Samuel CM. Rapid diagnosis of cryptococcal meningitis by use of lateral flow assay on cerebrospinal fluid samples: influence of the high-dose "hook" effect. J Clin Microbiol. 2014;52(12):4172-5.

12. Asawavichienjinda T, Sitthi-Amorn C, Tanyanont V. Serum cryptococcal antigen: diagnostic value in the diagnosis of AIDS-related cryptococcal meningitis. J Med Assoc Thailand=Chotmaihet Thangphaet. 1999;82(1):65-71.

13. Meya DB, Manabe YC, Castelnuovo B, Cook BA, Elbireer AM, Kambugu A, et al. Cost-effectiveness of serum cryptococcal antigen screening to prevent deaths among HIV-infected persons with a CD4 + cell count $<$ or $=100$ cells/microL who start HIV therapy in resource-limited settings. Clin Infect Dis Off Publ Infect Dis Soc Am. 2010;51(4):448-55.

14. Jarvis JN, Lawn SD, Vogt M, Bangani N, Wood R, Harrison TS. Screening for cryptococcal antigenemia in patients accessing an antiretroviral treatment program in South Africa. Clin Infect Dis. 2009;48(7):856-62.

15. Williams DA, Tadeo K, Velamakkani S, Kiggundu R, Meya DB, Rhein J, et al. Evaluating cryptococcal antigen lateral flow assay by fingerstick whole blood in HIV-infected persons with meningitis. IDweek; Oct. 11, 2014; Philadelphia, PA. Open Forum Infect Dis 2014.

16. Magambo KA, Kalluvya SE, Kapoor SW, Seni J, Chofle AA, Fitzgerald DW, et al. Utility of urine and serum lateral flow assays to determine the prevalence and predictors of cryptococcal antigenemia in HIV-positive outpatients beginning antiretroviral therapy in Mwanza. Tanzania J Int AIDS Soc. 2014;17:19040.

17. Kwizera R, Nguna J, Kiragga A, Nakavuma J, Rajasingham R, Boulware DR, et al. Performance of cryptococcal antigen lateral flow assay using saliva in Ugandans with CD4 $<100$. PLoS One. 2014;9(7):e103156.

18. Kabanda T, Siedner MJ, Klausner JD, Muzoora C, Boulware DR. Point-of-care diagnosis and prognostication of cryptococcal meningitis with the cryptococcal antigen lateral flow assay on cerebrospinal fluid. Clin Infect Dis. 2014;58(1):113-6.

19. Rhein J, Bahr NC, Morawski BM, Schutz C, Zhang Y, Finkelman $\mathrm{M}$, et al. Detection of high cerebrospinal fluid levels of (1->3)- -DGlucan in cryptococcal meningitis. Open Forum Infect Dis. 2014;1(3):ofu105-ofu.

20. Rhein J, Cloud JL, Hemmert AC, Bahr NC, Bellamkonda S, Oswald C, et al. Diagnostic performance of a multiplex PCR assay 
for meningitis in an HIV-infected population in Uganda poster abstract session. 2014

21. Bicanic T, Muzoora C, Brouwer AE, Meintjes G, Longley N, Taseera $\mathrm{K}$, et al. Independent association between rate of clearance of infection and clinical outcome of HIV-associated cryptococcal meningitis: analysis of a combined cohort of 262 patients. Clin Infect Dis Off Publ Infect Dis Soc Am. 2009;49(5):702-9.

22. Perfect JR, Dismukes WE, Dromer F, Goldman DL, Graybill JR, Hamill RJ, et al. Clinical practice guidelines for the management of cryptococcal disease: 2010 update by the infectious diseases society of America. Clin Infect Dis Off Publ Infect Dis Soc Am. 2010;50(3):291-322.

23.• Day JN, Chau TT, Wolbers M, Mai PP, Dung NT, Mai NH, et al. Combination antifungal therapy for cryptococcal meningitis. N Engl J Med. 2013;368(14):1291-302. Treatment with amphotericin $B$ and flucytosine improves survival in patients with cryptococcal meningitis.

24. Rajasingham R, Rolfes MA, Birkenkamp KE, Meya DB, Boulware DR. Cryptococcal meningitis treatment strategies in resourcelimited settings: a cost-effectiveness analysis. PLoS Med. 2012;9(9):e1001316.

25. Loyse A, Bicanic T, Jarvis JN. Combination antifungal therapy for cryptococcal meningitis. N Engl J Med. 2013;368(26):2522.

26. Loyse A, Thangaraj H, Easterbrook P, Ford N, Roy M, Chiller T, et al. Cryptococcal meningitis: improving access to essential antifungal medicines in resource-poor countries. Lancet Infect Dis. 2013;13(7):629-37.

27. Pappas PG, Chetchotisakd P, Larsen RA, Manosuthi W, Morris MI, Anekthananon T, et al. A phase II randomized trial of amphotericin $\mathrm{B}$ alone or combined with fluconazole in the treatment of HIVassociated cryptococcal meningitis. Clin Infect Dis Off Publ Infect Dis Soc Am. 2009;48(12):1775-83.

28. Loyse A, Wilson D, Meintjes G, Jarvis JN, Bicanic T, Bishop L, et al. Comparison of the early fungicidal activity of high-dose fluconazole, voriconazole, and flucytosine as second-line drugs given in combination with amphotericin B for the treatment of HIVassociated cryptococcal meningitis. Clin Infect Dis Off Publ Infect Dis Soc Am. 2012;54(1):121-8.

29. Muzoora CK, Kabanda T, Ortu G, Ssentamu J, Hearn P, Mwesigye $\mathrm{J}$, et al. Short course amphotericin B with high dose fluconazole for HIV-associated cryptococcal meningitis. J Infect. 2012;64(1):7681.

30. Jackson AT, Nussbaum JC, Phulusa J, Namarika D, Chikasema M, Kanyemba C, et al. A phase II randomized controlled trial adding oral flucytosine to high-dose fluconazole, with short-course amphotericin B, for cryptococcal meningitis. Aids. 2012;26(11): 1363-70.

31. Bahr NC, Rolfes MA, Musubire A, Nabeta H, Williams DA, Rhein $\mathrm{J}$, et al. Standardized electrolyte supplementation and fluid management improves survival during amphotericin therapy for cryptococcal meningitis in resource-limited settings. Open Forum Infect Dis. 2014;1(2):ofu070-ofu.

32. Govender NP, Patel J, van Wyk M, Chiller TM, Lockhart SR. Trends in antifungal drug susceptibility of Cryptococcus neoformans isolates obtained through population-based surveillance in South Africa in 2002-2003 and 2007-2008. Antimicrob Agents Chemother. 2011;55(6):2606-11.

33. Mdodo R, Moser SA, Jaoko W, Baddley J, Pappas P, Kempf MC, et al. Antifungal susceptibilities of Cryptococcus neoformans cerebrospinal fluid isolates from AIDS patients in Kenya. Mycoses. 2011;54(5):e438-42.

34. Jarvis JN, Meintjes G, Rebe K, Williams GN, Bicanic T, Williams A, et al. Adjunctive interferon-gamma immunotherapy for the treatment of HIV-associated cryptococcal meningitis: a randomized controlled trial. Aids. 2012;26(9):1105-13.
35. World Health Organization. Rapid advice: diagnosis, prevention and management of cryptococcal disease in HIV-infected adults, adolescents and children2011 May 31, 2012. Available from: http://www.who.int/hiv/pub/cryptococcal_disease2011.

36. Debruyne D. Clinical pharmacokinetics of fluconazole in superficial and systemic mycoses. Clin Pharmacokinet. 1997;33(1):52-77.

37. Rothe C, Sloan DJ, Goodson P, Chikafa J, Mukaka M, Denis B, et al. A prospective longitudinal study of the clinical outcomes from cryptococcal meningitis following treatment induction with $800 \mathrm{mg}$ oral fluconazole in Blantyre, Malawi. PloS One. 2013;8(6):e67311.

38. Longley N, Muzoora C, Taseera K, Mwesigye J, Rwebembera J, Chakera A, et al. Dose response effect of high-dose fluconazole for HIV-associated cryptococcal meningitis in southwestern Uganda. Clin Infect Dis Off Publ Infect Dis Soc Am. 2008;47(12):1556-61.

39. Bicanic T, Meintjes G, Wood R, Hayes M, Rebe K, Bekker LG, et al. Fungal burden, early fungicidal activity, and outcome in cryptococcal meningitis in antiretroviral-naive or antiretroviralexperienced patients treated with amphotericin B or fluconazole. Clin Infect Dis Off Publ Infect Dis Soc Am. 2007;45(1):76-80.

40. Bozzette SA, Larsen RA, Chiu J, Leal MA, Jacobsen J, Rothman P, et al. A placebo-controlled trial of maintenance therapy with fluconazole after treatment of cryptococcal meningitis in the acquired immunodeficiency syndrome. California Collaborative Treatment Group. New England J Med. 1991;324(9):580-4.

41. Saag MS, Powderly WG, Cloud GA, Robinson P, Grieco MH, Sharkey PK, et al. Comparison of amphotericin B with fluconazole in the treatment of acute AIDS-associated cryptococcal meningitis. N Engl J Med. 1992;326(2):83-9.

42. Saag MS, Cloud GA, Graybill JR, Sobel JD, Tuazon CU, Johnson $\mathrm{PC}$, et al. A comparison of itraconazole versus fluconazole as maintenance therapy for AIDS-associated cryptococcal meningitis. National Institute of Allergy and Infectious Diseases Mycoses Study Group. Clin Infect Dis. 1999;28(2):291-6.

43. Khoo SH, Bond J, Denning DW. Administering amphotericin B-a practical approach. J Antimicrob Chemother. 1994;33(2):203-13.

44. Sudan A, Livermore J, Howard SJ, Al-Nakeeb Z, Sharp A, Goodwin J, et al. Pharmacokinetics and pharmacodynamics of fluconazole for cryptococcal meningoencephalitis: implications for antifungal therapy and in vitro susceptibility breakpoints. Antimicrob Agents Chemother. 2013;57(6):2793-800.

45.• Boulware DR, Meya DB, Muzoora C, Rolfes MA, Huppler Hullsiek K, Musubire A, et al. Timing of antiretroviral therapy after diagnosis of cryptococcal meningitis. N Engl J Med. 2014;370(26): 2487-98. Deferred initiation of ART is associated with improved survival compared to early initiation of ART.

46. Krysan DJ. Toward improved anti-cryptococcal drugs: novel molecules and repurposed drugs. Fungal Genet Biol FG \& B. 2014.

47. Zhai B, Wu C, Wang L, Sachs MS, Lin X. The antidepressant sertraline provides a promising therapeutic option for neurotropic cryptococcal infections. Antimicrob Agents Chemother. 2012;56(7):3758-66.

48. Lewis RJ, Angier MK, Williamson KS, Johnson RD. Analysis of sertraline in postmortem fluids and tissues in 11 aviation accident victims. J Anal Toxicol. 2013;37(4):208-16.

49. Rhein J, Huppler Hullsiek K, Morawski BM, Smith KD, Al-Hadab A, Musubire A, et al. Adjunctive sertraline for the treatment of HIVassociated cryptococcal meningitis. Conference on Retroviruses and Opportunistic Infections (CROI); Feb. 26; Seattle, WA2015.

50. Loyse A, Wainwright H, Jarvis JN, Bicanic T, Rebe K, Meintjes G, et al. Histopathology of the arachnoid granulations and brain in HIV-associated cryptococcal meningitis: correlation with cerebrospinal fluid pressure. Aids. 2010;24(3):405-10.

51. Robertson EJ, Najjuka G, Rolfes MA, Akampurira A, Jain N, Anantharanjit J, et al. Cryptococcus neoformans ex vivo capsule size is associated with intracranial pressure and host immune 
response in HIV-associated cryptococcal meningitis. J Infect Dis. 2014;209(1):74-82.

52. Harris JR, Lockhart SR, Debess E, Marsden-Haug N, Goldoft M, Wohrle R, et al. Cryptococcus gattii in the United States: clinical aspects of infection with an emerging pathogen. Clin Infect Dis Off Publ Infect Dis Soc Am. 2011;53(12):1188-95.

53. Smith RM, Mba-Jonas A, Tourdjman M, Schimek T, DeBess E, Marsden-Haug N, et al. Treatment and outcomes among patients with Cryptococcus gattii infections in the United States Pacific Northwest. PLoS One. 2014;9(2):e88875.

54. Johnston SR, Corbett EL, Foster O, Ash S, Cohen J. Raised intracranial pressure and visual complications in AIDS patients with cryptococcal meningitis. J Infect. 1992;24(2):185-9.

55. Fessler RD, Sobel J, Guyot L, Crane L, Vazquez J, Szuba MJ, et al. Management of elevated intracranial pressure with cryptococcal meningitis. J Acquir Immune Defic Syndr Hum Retrovirol. 1997;17(2): 137-42.

56. Graybill JR, Sobel J, Saag M, van Der Horst C, Powderly W, Cloud $\mathrm{G}$, et al. Diagnosis and management of increased intracranial pressure in patients with AIDS and cryptococcal meningitis. Clin Infect Dis Off Publ Infect Dis Soc Am. 2000;30(1):47-54.

57. Rolfes MA, Hullsiek KH, Rhein J, Nabeta HW, Taseera K, Schutz $\mathrm{C}$, et al. The effect of therapeutic lumbar punctures on acute mortality from cryptococcal meningitis. Clin Infect Dis. 2014;59(11): 1607-14. Therapeutic lumbar puncture, regardless of opening pressure, improves survival.

58. Bicanic T, Brouwer AE, Meintjes G, Rebe K, Limmathurotsakul D, Chierakul W, et al. Relationship of cerebrospinal fluid pressure, fungal burden and outcome in patients with cryptococcal meningitis undergoing serial lumbar punctures. Aids. 2009;23(6):701-6.

59. Southern African HIV, Clinicians ST. Guideline for the prevention, diagnosis and management of cryptococcal meningitis among HIVinfected persons: 2013 update. Southern African J HIV Med. 2013;14(2):76.

60. Jarvis JN, Bicanic T, Loyse A, Namarika D, Jackson A, Nussbaum JC, et al. Determinants of mortality in a combined cohort of 501 patients with HIV-associated Cryptococcal meningitis: implications for improving outcomes. Clin Infect Dis Off Publ Infect Dis Soc Am. 2014;58(5):736-45.

61. Meda J, Kalluvya S, Downs JA, Chofle AA, Seni J, Kidenya B, et al. Cryptococcal meningitis management in Tanzania with strict schedule of serial lumber punctures using intravenous tubing sets: an operational research study. J Acquir Immune Defic Syndr. 2014;66(2):e31-6.

62. Nabeta HW, Bahr NC, Rhein J, Fossland N, Kiragga AN, Meya DB, et al. Accuracy of noninvasive intraocular pressure or optic nerve sheath diameter measurements for predicting elevated intracranial pressure in cryptococcal meningitis. Open Forum Infect Dis. 2014;1(3):ofu093-ofu.

63. Corti M, Priarone M, Negroni R, Gilardi L, Castrelo J, Arechayala AI, et al. Ventriculoperitoneal shunts for treating increased intracranial pressure in cryptococcal meningitis with or without ventriculomegaly. Rev Soc Bras Med Trop. 2014;47(4):524-7.

64. Zolopa A, Andersen J, Powderly W, Sanchez A, Sanne I, Suckow C, et al. Early antiretroviral therapy reduces AIDS progression/ death in individuals with acute opportunistic infections: a multicenter randomized strategy trial. PLoS One. 2009;4(5):e5575.

65. Haddow LJ, Colebunders R, Meintjes G, Lawn SD, Elliott JH, Manabe YC, et al. Cryptococcal immune reconstitution inflammatory syndrome in HIV-1-infected individuals: proposed clinical case definitions. Lancet Infect Dis. 2010;10(11):791-802.

66. Longley N, Harrison TS, Jarvis JN. Cryptococcal immune reconstitution inflammatory syndrome. Curr Opin Infect Dis. 2013;26(1): 26-34.

67. Katchanov J, Blechschmidt C, Nielsen K, Branding G, Arasteh K, Tintelnot K, et al. Cryptococcal meningoencephalitis relapse after an eight-year delay: an interplay of infection and immune reconstitution. Int J STD AIDS. 2014.

68. Chang CC, Dorasamy AA, Gosnell BI, Elliott JH, Spelman T, Omarjee $\mathrm{S}$, et al. Clinical and mycological predictors of cryptococcosis-associated immune reconstitution inflammatory syndrome. Aids. 2013;27(13):2089-99.

69. Boulware DR, Meya DB, Bergemann TL, Wiesner DL, Rhein J, Musubire A, et al. Clinical features and serum biomarkers in HIV immune reconstitution inflammatory syndrome after cryptococcal meningitis: a prospective cohort study. PLoS Med. 2010;7(12): e1000384

70. Makadzange AT, Ndhlovu CE, Takarinda K, Reid M, Kurangwa M, Gona P, et al. Early versus delayed initiation of antiretroviral therapy for concurrent HIV infection and cryptococcal meningitis in sub-Saharan Africa. Clin Infect Dis Off Publ Infect Dis Soc Am. 2010;50(11):1532-8.

71. Bisson GP, Molefi M, Bellamy S, Thakur R, Steenhoff A, Tamuhla $\mathrm{N}$, et al. Early versus delayed antiretroviral therapy and cerebrospinal fluid fungal clearance in adults with HIV and cryptococcal meningitis. Clin Infect Dis Off Publ Infect Dis Soc Am. 2013;56(8): 1165-73.

72. Boulware DR, Bonham SC, Meya DB, Wiesner DL, Park GS, Kambugu A, et al. Paucity of initial cerebrospinal fluid inflammation in cryptococcal meningitis is associated with subsequent immune reconstitution inflammatory syndrome. J Infect Dis. 2010;202(6):962-70.

73. Rhein J, Huppler Hullsiek K, Bahr NC, Kiggundu R, Williams DA, Nabeta HW, et al. Detrimental outcomes of unmasking cryptococcal meningitis with recent art initiation. Conference on Retroviruses and Opportunistic Infections (CROI); Feb. 26; Seattle, WA2015.

74. Jarvis JN, Meintjes G, Harrison TS. Outcomes of cryptococcal meningitis in antiretroviral naive and experienced patients in South Africa. J Infect. 2010;60(6):496-8.

75. Rajasingham R, Meya DB, Boulware DR. Integrating cryptococcal antigen screening and pre-emptive treatment into routine HIV care. J Acquir Immune Defic Syndr. 2012;59(5):e85-91.

76. Musubire AK, Boulware DR, Meya DB, Rhein J. Diagnosis and management of cryptococcal relapse. J AIDS Clin Res. 2013;3(Suppl 3)

77. Gamaletsou MN, Sipsas NV, Kontoyiannis DP, Tsiakalos A, Kontos AN, Stefanou I, et al. Successful salvage therapy of refractory HIV-related cryptococcal meningitis with the combination of liposomal amphotericin B, voriconazole, and recombinant interferon-gamma. Diagn Microbiol Infect Dis. 2012;74(4):409-11.

78. Brunel AS, Reynes J, Tuaillon E, Rubbo PA, Lortholary O, Montes $\mathrm{B}$, et al. Thalidomide for steroid-dependent immune reconstitution inflammatory syndromes during AIDS. Aids. 2012;26(16):2110-2.

79. Sitapati AM, Kao CL, Cachay ER, Masoumi H, Wallis RS, Mathews WC. Treatment of HIV-related inflammatory cerebral cryptococcoma with adalimumab. Clin Infect Dis Off Publ Infect Dis Soc Am. 2010;50(2):e7-10. 